\title{
Synthesis and Antibacterial Evaluation of 2-(1,3- Benzodioxol-5-ylcarbonyl)arylsulfonohydrazide Derivatives
}

\author{
Asia Siddiqa ${ }^{1}$, Aziz-ur-Rehman ${ }^{1 *}$, Muhammad Athar Abbasi ${ }^{1}$, Shahid Rasool ${ }^{1}$, \\ Khalid Mohammed Khan ${ }^{2}$, Irshad Ahmad ${ }^{3}$ and Saira Afzal ${ }^{3}$ \\ ${ }^{1}$ Department of Chemistry, Government College University, Lahore-54000, ${ }^{2}$ HEJ Research Institute of Chemistry, International \\ Center for Chemical and Biological Sciences, University of Karachi, Karachi-75270, ${ }^{3}$ Department of Pharmacy, The Islamia \\ University of Bahawalpur, Bahawalpur-63100, Pakistan
}

*For correspondence: Email: azizryk@yahoo.com, rehman@gcu.edu.pk; Tel: (+92)-42-111000010 ext 450

Received: 19 December 2013

Revised accepted: 22 August 2014

\begin{abstract}
Purpose: To study the antibacterial activity of various sulfonamides derived from 1,3-benzodioxol-5carbohydrazide.

Methods: The synthesis involved the conversion of 1,3-benzodioxol-5-carboxylic acid (1) to ethyl 1,3benzodioxol-5-carboxylate (2) and then to 1,3-benzodioxol-5-carbohydrazide (3). The target molecules, 2-(1,3-benzodioxol-5-ylcarbonyl)arylsulfonohydrazide derivatives (5a-l) were synthesized through a benignant method from aqueous medium by the reaction of 3 and arylsulfonyl chlorides (4a-l). The structural formulae of the synthesized compounds were characterized by infra red spectroscopy (IR), proton nuclear magnetic resonance $\left({ }^{1} \mathrm{H}-\mathrm{NMR}\right)$ and electron impact mass spectrometry (EI-MS). The compounds were screened for in vitro antibacterial activity by determining their minimum inhibitory concentration (MIC).

Results: The molecule, $5 k$, bearing 2-hydroxy-3,5-dichlorophenyl group exhibited the highest activity with MIC of $11.92 \pm 3.40$ (S. typhi), $8.37 \pm 2.22$ (E. coli), $9.28 \pm 2.31$ ( $P$. aeroginosa), $11.76 \pm 1.30$ (B. subtilis) and $10.30 \pm 1.63$ (S. aureus) $\mu$ moles/L relative to that of ciprofloxacin with $9.42 \pm 1.09,8.02 \pm$ $2.17,8.11 \pm 1.32,8.88 \pm 2.00$ and $9.23 \pm 1.87 \mu$ moles/L respectively.

Conclusion: The most potent of the synthesized compounds $(5 \mathrm{k})$ posesses moderate activity against all the bacterial strains, while $5 \mathrm{~g}$ remained completely inactive.
\end{abstract}

Keywords: 1,3-Benzodioxol-5-carboxylic acid, Antibacterial activity, Sulfonohydrazide, Synthesis

Tropical Journal of Pharmaceutical Research is indexed by Science Citation Index (SciSearch), Scopus, International Pharmaceutical Abstract, Chemical Abstracts, Embase, Index Copernicus, EBSCO, African Index Medicus, JournalSeek, Journal Citation Reports/Science Edition, Directory of Open Access Journals (DOAJ), African Journal Online, Bioline International, Open-J-Gate and Pharmacy Abstracts

\section{INTRODUCTION}

Most of the biologically active compounds include sulfonamides, and they are an important class of drugs showing various activities such as antimicrobial, antitumor, anticonvulsant, anticancer, anti-inflammatory, antidiuretic, antihydrod, insulin-releasing and hypoglycemic. They also act as inhibitors, e.g., histone deacetylase (HDAC), carbonic anhydrase and HIV protease [1-8]. The chemotherapeutic derivatives of this class are extensively employed as antibacterial and antiviral agents [4,9] since they are structural analogues of $p$-aminobenzoic acid necessary for the production of folic acid. If they are substituted for $p$-aminobenzoic acid, the production of folic acid stops and ultimately bacterial growth stops $[2,10]$.

In continuation of our earlier work on this class of compounds [11], we sought to synthesize new 2(1,3-benzodioxol-5-ylcarbonyl) 
arylsulfonohydrazide derivatives to evaluate antibacterial activity.

\section{EXPERIMENTAL}

\section{General}

Melting points of all the synthesized compounds were recorded on Griffin-George apparatus by open capillary tube and were uncorrected. Purity was confirmed Thin Layer Chromatography (TLC) on silica gel G-25-UV254 coated plates, run under varying ratios of solvent system of $n$ hexane and ethyl acetate. IR spectra were computed by $\mathrm{KBr}$ pellet method on a Jasco-320A spectrophotometer in $\mathrm{cm}^{-1}$. ${ }^{1} \mathrm{H}-\mathrm{NMR}$ spectra were measured in $\mathrm{CHCl}_{3}-\mathrm{d} 1$ on Bruker spectrometer at $300 / 400 \mathrm{MHz}$ and ${ }^{13} \mathrm{C}-\mathrm{NMR}$ at $100 \mathrm{MHz}$. Chemical shifts are provided in ppm with tetramethylsilane TMS as internal reference and coupling constant in $\mathrm{Hz}$. Mass spectra were recorded on a JMS-HX-110 spectrometer.

Procedure for the synthesis of ethyl 1,3benzodioxol-5-carboxylate (2)

The reaction mixture of 1,3-benzodioxol-5carboxylic acid $(1 ; 6.0 \mathrm{~g})$, ethanol $(24 \mathrm{~mL}, 99 \%)$ and concentrated $\mathrm{H}_{2} \mathrm{SO}_{4}(3.0 \mathrm{~mL})$ was set to reflux in $250 \mathrm{~mL}$ round bottom (RB) flask for $3 \mathrm{~h}$ till maximum completion, confirmed by TLC. The liquid ester (2) was extracted by solvent extraction at a $\mathrm{pH}$ of 8-10.

\section{Procedure for the synthesis of 1,3- benzodioxol-5-carbohydrazide (3)}

The reaction mixture of ethyl 1,3-benzodioxol-5carboxylate $(2 ; 0.2 \mathrm{~mol})$, ethanol $(150 \mathrm{~mL}, 99 \%)$ and hydrazine hydrate (15 mL, $80 \%)$ was refluxed in a round bottom flask for $6 \mathrm{~h}$. After completion of the reaction, ice cold distilled water was added to get solid precipitates of 3, which were filtered and washed with distilled water.

\section{Procedure for the synthesis of 2-(1,3- benzodioxol-5-ylcarbonyl) arylsulfonohydra- zide (5a-I)}

1,3-Benzodioxol-5-carbohydrazide (3; $0.005 \mathrm{~mol}$ ) was dispersed in $20 \mathrm{~mL}$ distilled water, adjusting the $\mathrm{pH}$ at 8-10 with sodium carbonate solution. Arylsulfonyl chlorides (4a-l; $0.005 \mathrm{~mol}$ ) were added gradually and the reaction mixture was stirred for 2-3 h. After the reaction was completed concentrated $\mathrm{HCl}(0.5 \mathrm{~mL})$ was added gradually while shaking. Precipitates of the products were obtained which were filtered, washed and dried.

\section{Antibacterial activity assay}

Antibacterial test was carried out in sterile 96well microplates under aseptic conditions. The method was based on the principle that microbial cell number increases as microbial growth proceeds in a log phase of growth, resulting in raised absorbance of broth medium $[12,13]$.

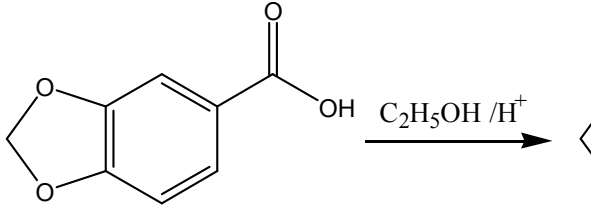

1<smiles>CCOC(=O)c1ccc2c(c1)OCO2</smiles>

2<smiles>NNC(=O)c1ccc2c(c1)OCO2</smiles>

3<smiles>[Y]C(C)C(=O)ON</smiles><smiles>[R]S(=O)(=O)NNC(=O)c1ccc2c(c1)OCO2</smiles>

5a-1 


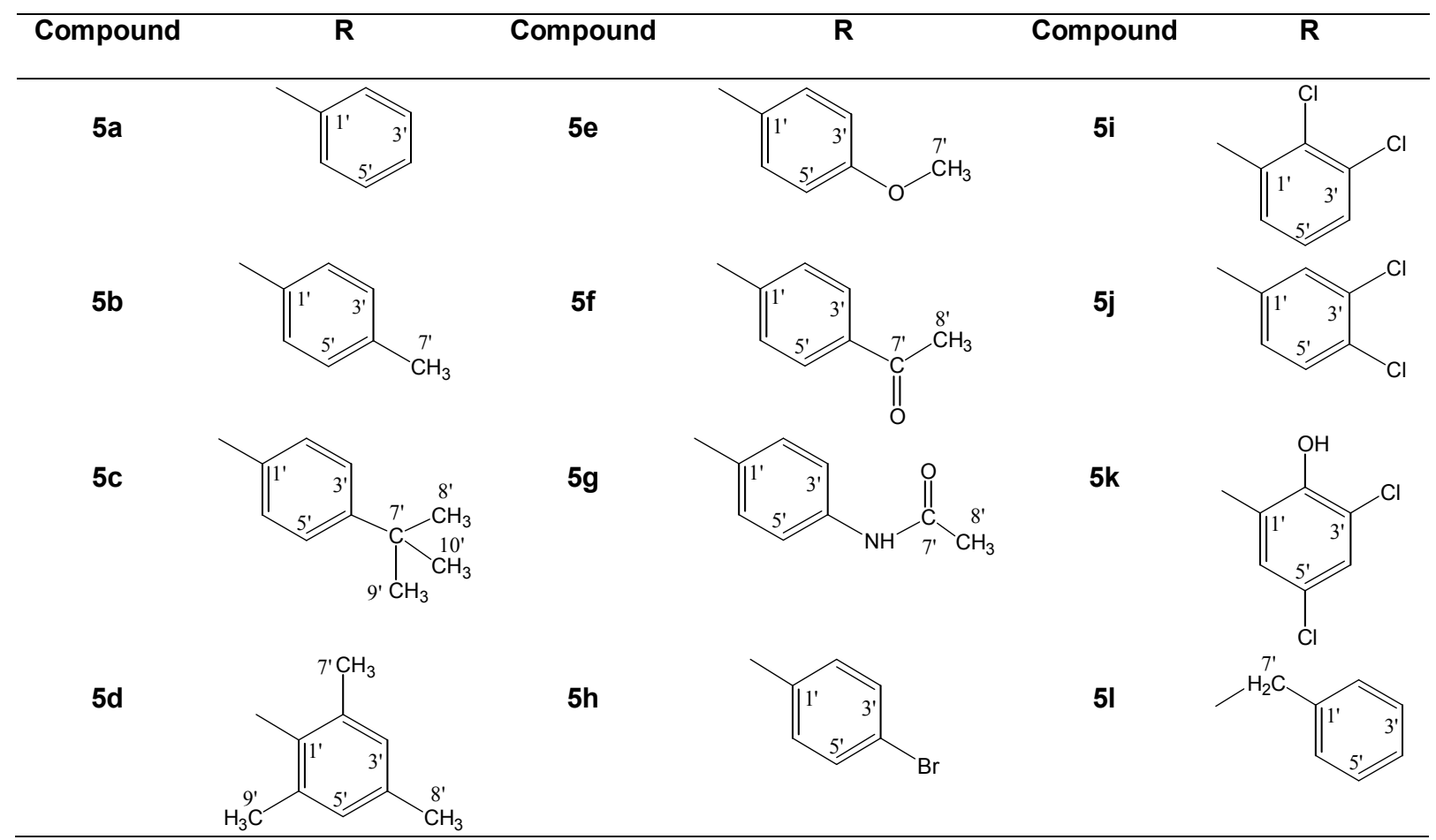

Scheme 1: Outline for synthesis of 2-(1,3-benzodioxol-5-ylcarbonyl)arylsulfonohydrazide derivatives (5a-I)

\section{RESULTS}

A new series of sulfonamides was synthesized from a facile method by scheme 1 . The synthesized 2-(1,3-benzodioxol-5ylcarbonyl)arylsulfonohydrazide derivatives (5a-I) were evaluated for antibacterial activity. The synthesized molecules were well supported by spectral data as follows and also the most of them remained moderately efficient against the bacterial strains taken into account.

\section{Chemistry}

The synthesis involved esterification of 1,3benzodioxol-5-carboxylic acid (1) to ethyl 1,3benzodioxol-5-carboxylate (2) by refluxing with ethanol and concentrated sulfuric acid. The ester was isolated using chloroform. The molecule, $\mathbf{2}$, was subjected to nucleophilic substitution reaction with hydrazine in ethanol to yield 1,3benzodioxol-5-carbohydrazide (3) which was treated with various arylsulfonyl chlorides in aqueous medium under dynamic $\mathrm{pH}$ control. The products were acquired through filtration from a slightly acidic medium. The low acidity of mixture is mandatory for better yields as higher has negative effect. The structures of all the synthesized molecules, 2, 3 and 5a-1, were corroborated through spectral data.

\section{Ethyl 1,3-benzodioxol-5-carboxylate (2)}

Yellowish transparent liquid; Yield: $90 \%$; Molecular formula: $\mathrm{C}_{10} \mathrm{H}_{10} \mathrm{O}_{4}$; Molecular mass: 194; IR ( $\mathrm{NaCl}$ discs): $v_{\max }\left(\mathrm{cm}^{-1}\right): 3006$ (aromatic $\mathrm{C}-\mathrm{H}$ stretching), 1710 ( $\mathrm{C}=\mathrm{O}$ ester stretching), 1605 (Ar C=C stretching), 1110 (C-O ester stretching); ${ }^{1} \mathrm{H}-\mathrm{NMR}\left(\mathrm{CDCl}_{3}, 400 \mathrm{MHz}, \delta / \mathrm{ppm}\right)$ : $7.64(\mathrm{dd}, \mathrm{J}=8.4,1.6 \mathrm{~Hz}, 1 \mathrm{H}, \mathrm{H}-6), 7.44$ (d, J = $1.2 \mathrm{~Hz}, 1 \mathrm{H}, \mathrm{H}-2), 6.82(\mathrm{~d}, \mathrm{~J}=8.0 \mathrm{~Hz}, 1 \mathrm{H}, \mathrm{H}-5)$, $6.01(\mathrm{~s}, 2 \mathrm{H}, \mathrm{H}-7), 4.32(\mathrm{q}, \mathrm{J}=7.2 \mathrm{~Hz}, 2 \mathrm{H}$, $\left.\mathrm{OCH}_{2} \mathrm{CH}_{3}\right), 1.37$ (t, J = 7.2 Hz, 3H, $-\mathrm{OCH}_{2} \mathrm{CH}_{3}$ ); ${ }^{13} \mathrm{C}-N M R\left(\mathrm{CDCl}_{3}, 100 \mathrm{MHz}, \mathrm{\delta} / \mathrm{ppm}\right): 165.4$ (C-8), 148.7 (C-4), 145.1 (C-3), 128.6 (C-6), 121.3 (C1), 109.5 (C-2), 106.9 (C-5), 101.6 (C-7), 62.2 ($\left.\mathrm{OCH}_{2}\right), 13.9\left(\mathrm{CH}_{3}\right)$; EIMS (m/z): $194[\mathrm{M}]^{+}(2 \%)$, $165(23 \%), 149$ (100\%), 121 (26\%), 119 (6\%), $91(7 \%), 29(15 \%)$.

\section{1,3-Benzodioxol-5-carbohydrazide (3)}

White amorphous solid; Yield: $97 \%$; M.P: 170 ${ }^{\circ} \mathrm{C}$; Molecular formula: $\mathrm{C}_{8} \mathrm{H}_{8} \mathrm{~N}_{2} \mathrm{O}_{3}$; Molecular mass: 180; IR (KBr): $v_{\max }\left(\mathrm{cm}^{-1}\right): 3250(\mathrm{NH}$ stretching), 3010 (aromatic $\mathrm{C}-\mathrm{H}$ stretching), 1730 $(\mathrm{C}=\mathrm{O}$ amide stretching), $1610 \quad(\mathrm{Ar} \quad \mathrm{C}=\mathrm{C}$ stretching); ${ }^{1} \mathrm{H}-\mathrm{NMR}\left(\mathrm{CDCl}_{3}, 400 \mathrm{MHz}, \delta / \mathrm{ppm}\right)$ : $7.66(\mathrm{dd}, \mathrm{J}=8.4,1.6 \mathrm{~Hz}, 1 \mathrm{H}, \mathrm{H}-6), 7.45(\mathrm{~d}, \mathrm{~J}=$ $1.2 \mathrm{~Hz}, 1 \mathrm{H}, \mathrm{H}-2), 6.87(\mathrm{~d}, \mathrm{~J}=8.0 \mathrm{~Hz}, 1 \mathrm{H}, \mathrm{H}-5)$, $6.06(\mathrm{~s}, 2 \mathrm{H}, \mathrm{H}-7) ;{ }^{13} \mathrm{C}-\mathrm{NMR}\left(\mathrm{CDCl}_{3}, 100 \mathrm{MHz}\right.$, ठ/ppm): 170.2 (C-8), 148.9 (C-3), 148.1 (C-4), 124.8 (C-1), 122.3 (C-6), 109.1 (C-5), 107.2 (C2), 101.9 (C-7); $\operatorname{EIMS~(m/z):~} 180$ [M] ${ }^{+}(1 \%), 165$ 
(24\%), 149 (100\%), 121 (25\%), 119 (5 \%), 91 $(8 \%)$.

\section{2-(1,3-Benzodioxol-5- ylcarbonyl)benzenesulfonohydrazide (5a)}

White amorphous solid; Yield: 92 \%; M.P: 209$211{ }^{\circ} \mathrm{C}$; Molecular formula: $\mathrm{C}_{14} \mathrm{H}_{12} \mathrm{~N}_{2} \mathrm{O}_{5} \mathrm{~S}$; Molecular mass: 320 ; IR $(\mathrm{KBr}): \mathrm{v}_{\max }\left(\mathrm{cm}^{-1}\right): 3256$ ( $\mathrm{NH}$ stretching), 3018 (aromatic $\mathrm{C}-\mathrm{H}$ stretching), 1733 ( $\mathrm{C}=\mathrm{O}$ amide stretching), 1612 ( $\mathrm{Ar} \mathrm{C}=\mathrm{C}$ stretching), 1413 ( $\mathrm{S}=\mathrm{O}$ stretching); ${ }^{1} \mathrm{H}-\mathrm{NMR}$ $\left(\mathrm{CDCl}_{3}, 400 \mathrm{MHz}, \delta / \mathrm{ppm}\right): 7.92(\mathrm{~d}, \mathrm{~J}=7.6 \mathrm{~Hz}$, $\left.2 \mathrm{H}, \mathrm{H}-2^{\prime} \& 6^{\prime}\right), 7.58\left(\mathrm{t}, \mathrm{J}=7.6 \mathrm{~Hz}, 1 \mathrm{H}, \mathrm{H}-4^{\prime}\right), 7.47$ (t, J = 7.2 Hz, 2H, H-3', 5'), 7.19 (dd, J = 8.4, 1.6 $\mathrm{Hz}, 1 \mathrm{H}, \mathrm{H}-6), 7.04$ (d, J = 1.2 Hz, 1H, H-2), 6.80 (d, J = 8.0 Hz, 1H, H-5), $6.01(\mathrm{~s}, 2 \mathrm{H}, \mathrm{H}-7) ;{ }^{13} \mathrm{C}-$ NMR $\left(\mathrm{CDCl}_{3}, 100 \mathrm{MHz}, \delta / \mathrm{ppm}\right): 170.5$ (C-8), 148.7 (C-4), 146.4 (C-3), 145.8 (C-1'), 132.4 (C4'), 127.1 (C-3', C-5'), 125.6 (C-1), 124.8 (C-2', C-6'), 122.6 (C-6), 109.3 (C-2), 107.4 (C-5), 101.6 (C-7); EIMS $(\mathrm{m} / \mathrm{z}): 320\left[\mathrm{M}^{+}(1 \%), 149\right.$ (100\%), 121 (27\%), 91 (7\%).

\section{2-(1,3-Benzodioxol-5-ylcarbonyl)-4- methylbenzenesulfonohydrazide (5b)}

White amorphous solid; Yield: $90 \%$; M.P: 204$206{ }^{\circ} \mathrm{C}$; Molecular formula: $\mathrm{C}_{15} \mathrm{H}_{14} \mathrm{~N}_{2} \mathrm{O}_{5} \mathrm{~S}$; Molecular mass: 334 ; IR (KBr): $v_{\max }\left(\mathrm{cm}^{-1}\right): 3259$ ( $\mathrm{NH}$ stretching), 3027 (aromatic $\mathrm{C}-\mathrm{H}$ stretching), 1739 ( $\mathrm{C}=\mathrm{O}$ amide stretching), 1617 (Ar C=C stretching), 1418 ( $\mathrm{S}=\mathrm{O}$ stretching); ${ }^{1} \mathrm{H}-\mathrm{NMR}$ $\left(\mathrm{CDCl}_{3}, 400 \mathrm{MHz}, \delta / \mathrm{ppm}\right): 7.79(\mathrm{~d}, \mathrm{~J}=8.0 \mathrm{~Hz}$, $\left.2 \mathrm{H}, \mathrm{H}-2,{ }^{\prime} 6^{\prime}\right), 7.42\left(\mathrm{~d}, \mathrm{~J}=7.6 \mathrm{~Hz}, 2 \mathrm{H}, \mathrm{H}-3,{ }^{\prime} 5^{\prime}\right)$, $7.19(\mathrm{dd}, \mathrm{J}=8.4,1.6 \mathrm{~Hz}, 1 \mathrm{H}, \mathrm{H}-6), 7.06(\mathrm{~d}, \mathrm{~J}=$ $1.6 \mathrm{~Hz}, 1 \mathrm{H}, \mathrm{H}-2), 6.81(\mathrm{~d}, \mathrm{~J}=8.4 \mathrm{~Hz}, 1 \mathrm{H}, \mathrm{H}-5)$, 6.01 (s, 2H, H-7), 2.35 (s, 3H, H-7'); ${ }^{13} \mathrm{C}-\mathrm{NMR}$ $\left(\mathrm{CDCl}_{3}, 100 \mathrm{MHz}, \delta / \mathrm{ppm}\right): 170.7$ (C-8), 148.4 (C4), 146.6 (C-3), 145.3 (C-1'), 132.9 (C-4'), 127.8 (C-2' C-6'), 125.2 (C-3' C-5'), 124.6 (C-1), 122.5 (C-6), 109.1 (C-2), 107.6 (C-5), 101.2 (C-7), 20.3 $\left(\mathrm{C}-7^{\prime}\right) ; \operatorname{EIMS}(\mathrm{m} / \mathrm{z}): 334$ [M] $^{+}(2 \%), 156(15 \%)$, $149(100 \%), 121(25 \%)$.

\section{2-(1,3-Benzodioxol-5-ylcarbonyl)-4-ter- butylbenzenesulfonohydrazide (5c)}

Brown amorphous solid; Yield: 96 \%; M.P: 259$261{ }^{\circ} \mathrm{C}$; Molecular formula: $\mathrm{C}_{18} \mathrm{H}_{20} \mathrm{~N}_{2} \mathrm{O}_{5} \mathrm{~S}$; Molecular mass: 376 ; IR $(\mathrm{KBr}): \mathrm{v}_{\max }\left(\mathrm{cm}^{-1}\right): 3272$ ( $\mathrm{NH}$ stretching), 3043 (aromatic $\mathrm{C}-\mathrm{H}$ stretching), 1733 ( $\mathrm{C}=\mathrm{O}$ amide stretching), 1612 ( $\mathrm{Ar} \mathrm{C}=\mathrm{C}$ stretching), 1407 ( $\mathrm{S}=\mathrm{O}$ stretching); ${ }^{1} \mathrm{H}-\mathrm{NMR}$ $\left(\mathrm{CDCl}_{3}, 300 \mathrm{MHz}, \delta / \mathrm{ppm}\right): 7.82(\mathrm{~d}, \mathrm{~J}=7.2 \mathrm{~Hz}$, $\left.2 \mathrm{H}, \mathrm{H}-2^{\prime} \& 6^{\prime}\right), 7.45$ (d, J = $\left.7.2 \mathrm{~Hz}, 2 \mathrm{H}, \mathrm{H}-3^{\prime} \& 5^{\prime}\right)$, $7.16(\mathrm{dd}, \mathrm{J}=7.2,1.5 \mathrm{~Hz}, 1 \mathrm{H}, \mathrm{H}-6), 7.04(\mathrm{~d}, \mathrm{~J}=$ $1.5 \mathrm{~Hz}, 1 \mathrm{H}, \mathrm{H}-2), 6.80(\mathrm{~d}, \mathrm{~J}=7.2 \mathrm{~Hz}, 1 \mathrm{H}, \mathrm{H}-5)$, 1.35 (s, 9H, H-8' to $\left.10^{\prime}\right) ;{ }^{13} \mathrm{C}-\mathrm{NMR}\left(\mathrm{CDCl}_{3}, 100\right.$ $\mathrm{MHz}, \quad \delta / p p m): 170.4$ (C-8), 152.8 (C-4'), 148.1
(C-4), 146.7 (C-3), 145.6 (C-1'), 126.9 (C-2' C6'), 125.3 (C-3' C-5'), 124.9 (C-1), 122.4 (C-6), 109.8 (C-2), 107.2 (C-5), 101.5 (C-7), 32.3 (C-7'), 31.1 (C-8' to C-10'); EIMS (m/z): $376\left[\mathrm{M}^{+}(1 \%)\right.$, $331(5 \%), 316(2 \%), 198(20 \%), 149(100 \%)$, $121(25 \%), 119(6 \%)$.

\section{2-(1,3-Benzodioxol-5-ylcarbonyl)mesitylen-2- ylsulfonohydrazide (5d)}

Creamy white amorphous solid; Yield: $96 \%$; M.P: $269-271{ }^{\circ} \mathrm{C}$; $\quad$ Molecular formula: $\mathrm{C}_{17} \mathrm{H}_{18} \mathrm{~N}_{2} \mathrm{O}_{5} \mathrm{~S}$; Molecular mass: 362; IR (KBr): $\mathrm{V}_{\max }\left(\mathrm{cm}^{-1}\right): 3278$ ( $\mathrm{NH}$ stretching), 3032 (aromatic $\mathrm{C}-\mathrm{H}$ stretching), 1737 ( $\mathrm{C}=\mathrm{O}$ amide stretching), 1607 ( $\mathrm{Ar} \mathrm{C}=\mathrm{C}$ stretching), 1395 ( $\mathrm{S}=\mathrm{O}$ stretching); ${ }^{1} \mathrm{H}-\mathrm{NMR}\left(\mathrm{CDCl}_{3}, 400 \mathrm{MHz}, \delta / \mathrm{ppm}\right): 7.17$ (d, $\mathrm{J}=$ $7.6 \mathrm{~Hz}, 1 \mathrm{H}, \mathrm{H}-6), 7.06$ (s, 1H, H-2), 6.89 (s, 2H, H-3' 5'), 6.79 (d, J = 8.0 Hz, 1H, H-5), $6.00(\mathrm{~s}$, $2 \mathrm{H}, \mathrm{H}-7$ ), 2.71 (s, 6H, H-7' 9'), 2.23 (s, 3H, H-8'); ${ }^{13} \mathrm{C}-\mathrm{NMR}\left(\mathrm{CDCl}_{3}, 100 \mathrm{MHz}, \delta / \mathrm{ppm}\right): 170.7$ (C-8), 152.3 (C-1'), 149.7 (C-2' \& C-6'), 148.2 (C-4), 146.4 (C-3), 140.1 (C-4'), 139.5 (C-3' \& C-5'), 124.4 (C-1), 122.7 (C-6), 109.3 (C-2), 107.9 (C5), 101.1 (C-7), 23.1 (C-7' \& C-9'), 20.2 (C-8'); EIMS (m/z): $362[\mathrm{M}]^{+}(1 \%), 184(15 \%), 165(30$ $\%), 149(100 \%), 121(22 \%)$.

\section{2-(1,3-Benzodioxol-5-ylcarbonyl)-4- methoxybenzenesulfonohydrazide (5e)}

White amorphous solid; Yield: 87 \%; M.P: 319$321{ }^{\circ} \mathrm{C}$; Molecular formula: $\mathrm{C}_{15} \mathrm{H}_{14} \mathrm{~N}_{2} \mathrm{O}_{6} \mathrm{~S}$; Molecular mass: 350 ; IR $(\mathrm{KBr}): \mathrm{v}_{\max }\left(\mathrm{cm}^{-1}\right): 3279$ ( $\mathrm{NH}$ stretching), 3023 (aromatic $\mathrm{C}-\mathrm{H}$ stretching), 1739 ( $\mathrm{C}=\mathrm{O}$ amide stretching), 1626 ( $\mathrm{Ar} \mathrm{C}=\mathrm{C}$ stretching), 1419 ( $\mathrm{S}=\mathrm{O}$ stretching); ${ }^{1} \mathrm{H}-\mathrm{NMR}$ $\left(\mathrm{CDCl}_{3}, 300 \mathrm{MHz}, \delta / \mathrm{ppm}\right): 7.84(\mathrm{~d}, \mathrm{~J}=8.7 \mathrm{~Hz}$, $\left.2 \mathrm{H}, \mathrm{H}-2^{\prime}, 6^{\prime}\right), 7.21$ (dd, J = 9.3, $\left.1.2 \mathrm{~Hz}, 1 \mathrm{H}, \mathrm{H}-6\right)$, $7.07(\mathrm{~d}, \mathrm{~J}=1.5 \mathrm{~Hz}, 1 \mathrm{H}, \mathrm{H}-2), 6.90(\mathrm{~d}, \mathrm{~J}=9.0 \mathrm{~Hz}$, $\left.2 \mathrm{H}, \mathrm{H}^{\prime} 3^{\prime} \& 5^{\prime}\right), 6.80$ (d, J = 8.1 Hz, 1H, H-5), 6.01 (s, 2H, H-7), 3.80 (s, 3H, H-7'); ${ }^{13} \mathrm{C}-\mathrm{NMR}\left(\mathrm{CDCl}_{3}\right.$, $100 \mathrm{MHz}, \delta / \mathrm{ppm}): 170.5$ (C-8), 162.3 (C-4'), 148.7 (C-4), 146.4 (C-3), 144.1 (C-1'), 127.2 (C2', C-6'), 124.8 (C-1), 123.1 (C-6), 114.7 (C-3', C-5'), 109.4 (C-2), 107.4 (C-5), 101.6 (C-7), 57.8 (C-7'); EIMS (m/z): $350[\mathrm{M}]^{+}(50 \%), 172(59 \%)$, 165 (25\%), 149 (100\%), 121 (51\%), 107 (7\%), $91(9 \%)$.

\section{2-(1,3-Benzodioxol-5-ylcarbonyl)-4- acetylbenzenesulfonohydrazide (5f)}

Light brown amorphous solid; Yield: 97 \%; M.P: 199-201 ${ }^{\circ} \mathrm{C}$; Molecular formula: $\mathrm{C}_{16} \mathrm{H}_{14} \mathrm{~N}_{2} \mathrm{O}_{6} \mathrm{~S}$; Molecular mass: 362 ; IR $(\mathrm{KBr}): \mathrm{v}_{\max }\left(\mathrm{cm}^{-1}\right): 3265$ ( $\mathrm{NH}$ stretching), 3019 (aromatic $\mathrm{C}-\mathrm{H}$ stretching), 1739 ( $\mathrm{C}=\mathrm{O}$ amide stretching), 1629 (Ar C=C stretching), 1417 ( $\mathrm{S}=\mathrm{O}$ stretching); ${ }^{1} \mathrm{H}-\mathrm{NMR}$ 
$\left(\mathrm{CDCl}_{3}, 400 \mathrm{MHz}, \delta / p p m\right): 8.08(\mathrm{~d}, \mathrm{~J}=3.6 \mathrm{~Hz}$, $1 \mathrm{H}, \mathrm{H}-2), 7.89$ (d, J = 9.6 Hz, 2H, H-2' \& 6'), 7.57 (d, J = 8.0 Hz, 2H, H-3,' 5'), 7.37 (dd, J = 8.4, 1.6 $\mathrm{Hz}, 1 \mathrm{H}, \mathrm{H}-6), 6.75$ (d, J = $8.4 \mathrm{~Hz}, 1 \mathrm{H}, \mathrm{H}-5), 5.99$ (s, 2H, H-7), 2.60 (s, 3H, H-8'); ${ }^{13} \mathrm{C}-\mathrm{NMR}\left(\mathrm{CDCl}_{3}\right.$, $100 \mathrm{MHz}, \delta / p p m): 197.3$ (C-8'), 170.8 (C-8), 149.5 (C-1'), 148.2 (C-4), 146.9 (C-3), 137.3 (C4'), 126.6 (C-2', C-6'), 126.2 (C-3', C-5'), 125.7 (C-1), 123.3 (C-6), 109.6 (C-2), 107.7 (C-5), 101.4 (C-7), 25.8 (C-7'); EIMS (m/z): $362[\mathrm{M}]^{+}(1$ \%), 184 (12\%), 165 (28\%), 149 (100\%), 121 (28\%), 119 (6\%).

\section{2-(1,3-Benzodioxol-5-ylcarbonyl)-4- acetamidobenzenesulfonohydrazide $(5 \mathrm{~g})$}

Creamy white amorphous solid; Yield: $95 \%$; M.P: $249-251 \quad{ }^{\circ} \mathrm{C}$; $\quad$ Molecular formula: $\mathrm{C}_{16} \mathrm{H}_{15} \mathrm{~N}_{3} \mathrm{O}_{6} \mathrm{~S}$; Molecular mass: 377 ; IR (KBr): $\mathrm{V}_{\max }\left(\mathrm{cm}^{-1}\right)$ : 3267 (NH stretching), 3023 (aromatic $\mathrm{C}-\mathrm{H}$ stretching), 1741 ( $\mathrm{C}=\mathrm{O}$ amide stretching), 1603 (Ar C=C stretching), 1409 ( $\mathrm{S}=\mathrm{O}$ stretching); ${ }^{1} \mathrm{H}-\mathrm{NMR}\left(\mathrm{CDCl}_{3}, 400 \mathrm{MHz}, \delta / p p m\right): 7.73$ (d, J = $\left.8.8 \mathrm{~Hz}, 2 \mathrm{H}, \mathrm{H}-2^{\prime} \& 6^{\prime}\right), 7.58$ (d, J = 8.8 Hz, 2H, H3' \& 5'), 7.17 (dd, J = 8.4, 1.6 Hz, 1H, H-6), 7.03 $(\mathrm{d}, \mathrm{J}=1.6 \mathrm{~Hz}, 1 \mathrm{H}, \mathrm{H}-2), 6.72(\mathrm{~d}, \mathrm{~J}=8.0 \mathrm{~Hz}, 1 \mathrm{H}$, $\mathrm{H}-5), 5.93$ (s, 2H, H-7), 2.05 (s, 3H, H-8'); ${ }^{13} \mathrm{C}-$ NMR $\left(\mathrm{CDCl}_{3}, 100 \mathrm{MHz}, \delta / \mathrm{ppm}\right): 170.4$ (C-8), 167.8 (C-8'), 148.6 (C-4), 146.3 (C-3), 142.8 (C1'), 138.2 (C-4'), 125.5 (C-1), 124.2 (C-2,' C-6'), 123.7 (C-6), 112.4 (C-3', C-5'), 109.4 (C-2), 107.2 (C-5), 101.8 (C-7), 23.7 (C-7'); EIMS (m/z): $377 \mathrm{CM}^{+}(1 \%), 149(100 \%), 134(1.2 \%), 121$ (23\%), $119(4 \%), 91$ (8\%).

\section{2-(1,3-Benzodioxol-5-ylcarbonyl)-4- bromobenzenesulfonohydrazide (5h)}

Brown liquid; Yield: $89 \%$; Molecular formula: $\mathrm{C}_{14} \mathrm{H}_{11} \mathrm{BrN}_{2} \mathrm{O}_{5} \mathrm{~S}$; Molecular mass: 398; IR ( $\mathrm{NaCl}$ discs): $v_{\max }\left(\mathrm{cm}^{-1}\right): 3264$ ( $\mathrm{NH}$ stretching), 3046 (aromatic $\mathrm{C}-\mathrm{H}$ stretching), $1730 \quad(\mathrm{C}=\mathrm{O}$ amide stretching), 1601 ( $\mathrm{Ar} \mathrm{C}=\mathrm{C}$ stretching), 1398 (S=O stretching), 669 (C-Br stretching); ${ }^{1} \mathrm{H}-\mathrm{NMR}$ $\left(\mathrm{CDCl}_{3}, 400 \mathrm{MHz}, \delta / \mathrm{ppm}\right): 7.77(\mathrm{~d}, \mathrm{~J}=8.4 \mathrm{~Hz}$, $\left.2 \mathrm{H}, \mathrm{H}-2^{\prime} \& 6^{\prime}\right), 7.59$ (d, J = 8.8 Hz, 2H, H-3' \& 5'), 7.20 (dd, J = 8.4, $1.6 \mathrm{~Hz}, 1 \mathrm{H}, \mathrm{H}-6), 7.07$ (d, J = $1.2 \mathrm{~Hz}, 1 \mathrm{H}, \mathrm{H}-2), 6.82$ (d, J = $8.0 \mathrm{~Hz}, 1 \mathrm{H}, \mathrm{H}-5)$, 6.02 (s, 2H, H-7); ${ }^{13} \mathrm{C}-\mathrm{NMR}\left(\mathrm{CDCl}_{3}, 100 \mathrm{MHz}\right.$, ठ/ppm): 170.3 (C-8), 148.3 (C-4), 146.7 (C-3), 146.1 (C-1'), 130.4 (C-3' \& C-5'), 126.9 (C-2' \& C6'), 126.2 (C-4'), 125.5 (C-1), 123.8 (C-6), 109.7 (C-2), 107.3 (C-5), 101.5 (C-7); EIMS (m/z): 400 $[\mathrm{M}+2]^{+}(2 \%), 398\left[^{\mathrm{M}}\right]^{+}(2 \%), 222(22 \%), 220$ (22\%), 157 (15\%), 155 (15\%), 149 (100\%), $121(25 \%), 119$ (6\%), 91 (7\%).

\section{2-(1,3-Benzodioxol-5-ylcarbonyl)-2,3-} dichlorobenzenesulfonohydrazide (5i)

Yellow amorphous solid; Yield: 98 \%; M.P: 354$356{ }^{\circ} \mathrm{C}$; Molecular formula: $\mathrm{C}_{14} \mathrm{H}_{10} \mathrm{Cl}_{2} \mathrm{~N}_{2} \mathrm{O}_{5} \mathrm{~S}$; Molecular mass: 388; IR ( $\mathrm{KBr}): \mathrm{v}_{\max }\left(\mathrm{cm}^{-1}\right): 3286$ (NH stretching), 3028 (aromatic $\mathrm{C}-\mathrm{H}$ stretching), 1743 ( $\mathrm{C}=\mathrm{O}$ amide stretching), 1615 ( $\mathrm{Ar} \mathrm{C}=\mathrm{C}$ stretching), 1435 ( $\mathrm{S}=\mathrm{O}$ stretching), 703 (C-Cl stretching); ${ }^{1} \mathrm{H}-\mathrm{NMR}\left(\mathrm{CDCl}_{3}, 300 \mathrm{MHz}, \delta / p p m\right)$ : 7.97 (d, J = 7.8 Hz, 1H, H-6'), 7.81 (d, J = 7.5 Hz, $\left.1 \mathrm{H}, \mathrm{H}-5^{\prime}\right), 7.66$ (d, J = 7.5 Hz, 1H, H-4'), 7.18 (dd, $\mathrm{J}=7.5,2.1 \mathrm{~Hz}, 1 \mathrm{H}, \mathrm{H}-6), 7.06(\mathrm{~d}, \mathrm{~J}=2.4 \mathrm{~Hz}, 1 \mathrm{H}$, $\mathrm{H}-2), 6.79$ (d, J = 8.1 Hz, 1H, H-5), 5.99 (s, 2H, $\mathrm{H}-7) ;{ }^{13} \mathrm{C}-\mathrm{NMR}\left(\mathrm{CDCl}_{3}, 100 \mathrm{MHz}, \delta / \mathrm{ppm}\right): 170.6$ (C-8), 148.7 (C-4), 146.9 (C-3), 143.2 (C-1'), 134.1 (C-2'), 132.8 (C-4'), 131.4 (C-3'), 126.7 (C5'), 125.4 (C-1), 124.5 (C-6'), 123.6 (C-6), 109.9 (C-2), 107.1 (C-5), 101.6 (C-7); EIMS (m/z): 392 $[\mathrm{M}+4]^{+}(0.7 \%), 390[\mathrm{M}+2]^{+}(1 \%), 388[\mathrm{M}]^{+}(2 \%)$, 213 (5\%), 211 (8\%), 209 (25\%), 165 (25\%), 149 (100\%), 147 (30\%), 145 (60\%), 123 (2\%), 119 (5\%).

\section{2-(1,3-Benzodioxol-5-ylcarbonyl)-3,4- dichlorobenzenesulfonohydrazide (5j)}

White amorphous solid; Yield: $98 \%$; M.P: 399$401{ }^{\circ} \mathrm{C}$; Molecular formula: $\mathrm{C}_{14} \mathrm{H}_{10} \mathrm{Cl}_{2} \mathrm{~N}_{2} \mathrm{O}_{5} \mathrm{~S}$; Molecular mass: 388; IR ( $\mathrm{KBr}): \mathrm{v}_{\max }\left(\mathrm{cm}^{-1}\right): 3275$ (NH stretching), 3036 (aromatic $\mathrm{C}-\mathrm{H}$ stretching), 1741 ( $\mathrm{C}=\mathrm{O}$ amide stretching), 1605 ( $\mathrm{Ar} \mathrm{C}=\mathrm{C}$ stretching), 1415 ( $\mathrm{S}=\mathrm{O}$ stretching), 707 (C-Cl stretching); ${ }^{1} \mathrm{H}-\mathrm{NMR}\left(\mathrm{CDCl}_{3}, 300 \mathrm{MHz}, \delta / \mathrm{ppm}\right)$ : 7.98 (s, 1H, H-2'), 7.73 (d, J = 7.5 Hz, 1H, H-6'), 7.53 (d, J = 8.4 Hz, 1H, H-5'), 7.19 (dd, J = 7.8, $2.1 \mathrm{~Hz}, 1 \mathrm{H}, \mathrm{H}-6), 7.06$ (d, J = $2.4 \mathrm{~Hz}, 1 \mathrm{H}, \mathrm{H}-2)$, $6.83(\mathrm{~d}, \mathrm{~J}=8.1 \mathrm{~Hz}, 1 \mathrm{H}, \mathrm{H}-5), 6.03$ (s, 2H, H-7); ${ }^{13} \mathrm{C}-\mathrm{NMR}\left(\mathrm{CDCl}_{3}, 100 \mathrm{MHz}, \delta / \mathrm{ppm}\right): 170.8$ (C-8), 148.5 (C-4), 146.8 (C-3), 142.9 (C-1'), 132.2 (C4'), 128.6 (C-5'), 127.5 (C-3'), 126.2 (C-2'), 125.6 (C-1), 124.6 (C-6'), 123.3 (C-6), 109.7 (C-2), 107.4 (C-5), 101.9 (C-7); EIMS (m/z): $392[\mathrm{M}+4]^{+}$ $(0.7 \%), 390[\mathrm{M}+2]^{+}(1 \%), 388[\mathrm{M}]^{+}(2 \%), 213(9$ \%), 211 (13\%), 209 (28\%), 165 (30\%), 149 (100\%), 147 (32\%), 145 (63\%), 123 (2\%), 119 (5\%).

\section{2-(1,3-Benzodioxol-5-ylcarbonyl)-3,5-dichloro- 2-hydroxybenzenesulfonohydrazide (5k)}

Light brown amorphous solid; Yield: 92 \%; M.P: 188-190 ${ }^{\circ} \mathrm{C}$; Molecular formula: $\mathrm{C}_{14} \mathrm{H}_{10} \mathrm{Cl}_{2} \mathrm{~N}_{2} \mathrm{O}_{6} \mathrm{~S}$; Molecular mass: 404; IR ( $\mathrm{KBr}): \mathrm{v}_{\max }\left(\mathrm{cm}^{-1}\right): 3276$ (NH stretching), 3016 (aromatic $\mathrm{C}-\mathrm{H}$ stretching), 1735 ( $\mathrm{C}=\mathrm{O}$ amide stretching), 1608 ( $\mathrm{Ar} \mathrm{C}=\mathrm{C}$ stretching), 1401 ( $\mathrm{S}=\mathrm{O}$ stretching), 701 (C-Cl stretching); ${ }^{1} \mathrm{H}-\mathrm{NMR}\left(\mathrm{CDCl}_{3}, 400 \mathrm{MHz}, \delta / p p m\right)$ : 
7.57 (d, J = 2.8 Hz, 1H, H-6'), 7.41 (d, J = 2.8 Hz, $1 \mathrm{H}, \mathrm{H}-4$ ') 7.17 (dd, J = 8.4, $1.6 \mathrm{~Hz}, 1 \mathrm{H}, \mathrm{H}-6)$, $7.05(\mathrm{~d}, \mathrm{~J}=1.6 \mathrm{~Hz}, 1 \mathrm{H}, \mathrm{H}-2), 6.73(\mathrm{~d}, \mathrm{~J}=8.0 \mathrm{~Hz}$, $1 \mathrm{H}, \mathrm{H}-5), 5.95$ (s, 2H, H-7); ${ }^{13} \mathrm{C}-\mathrm{NMR}\left(\mathrm{CDCl}_{3}\right.$, $100 \mathrm{MHz}, \delta / p p m): 170.4$ (C-8), 148.4 (C-4), 146.7 (C-3), 143.2 (C-1'), 133.6 (C-3' C-5'), 129.4 (C-4'), 125.5 (C-1), 124.1 (C-2' C-6'), 123.5 (C6), 109.4 (C-2), 107.7 (C-5), 101.8 (C-7); EIMS $(\mathrm{m} / \mathrm{z}): 408[\mathrm{M}+4]^{+}(0.7 \%), 406[\mathrm{M}+2]^{+}(1 \%), 404$ [M] $^{+}(2 \%), 226(24 \%), 165(25 \%), 162(90 \%)$, $149(100 \%), 119(5 \%)$.

\section{2-(1,3-Benzodioxol-5- ylcarbonyl)benzylsulfonohydrazide (5I)}

White amorphous solid; Yield: $87 \%$; M.P: 274$276{ }^{\circ} \mathrm{C}$; Molecular formula: $\mathrm{C}_{15} \mathrm{H}_{14} \mathrm{~N}_{2} \mathrm{O}_{5} \mathrm{~S}$; Molecular mass: 334 ; IR (KBr): $v_{\max }(\mathrm{cm}-1)$ : 3273 (NH stretching), 3024 (aromatic C-H stretching), 1746 ( $\mathrm{C}=\mathrm{O}$ amide stretching), 1614 ( $\mathrm{Ar} \mathrm{C}=\mathrm{C}$ stretching), 1411 ( $\mathrm{S}=\mathrm{O}$ stretching); ${ }^{1} \mathrm{H}-\mathrm{NMR}$ $\left(\mathrm{CDCl}_{3}, 400 \mathrm{MHz}, \delta / p p m\right):$ 7.47-7.35 (m, 5H, H-2' to $\left.6^{\prime}\right), 7.32$ (dd, J = 8.0, $\left.1.6 \mathrm{~Hz}, 1 \mathrm{H}, \mathrm{H}-6\right), 7.08$ (d, $\mathrm{J}=2.4 \mathrm{~Hz}, 1 \mathrm{H}, \mathrm{H}-2), 6.86(\mathrm{~d}, \mathrm{~J}=8.0 \mathrm{~Hz}, 1 \mathrm{H}, \mathrm{H}-$
5), 6.05 (s, 2H, H-7), 4.37 (s, 2H, H-7'); ${ }^{13} \mathrm{C}-\mathrm{NMR}$ $\left(\mathrm{CDCl}_{3}, 100 \mathrm{MHz}, \delta / p p m\right): 170.7$ (C-8), 148.2 (C4), 146.5 (C-3), 130.4 (C-2' C-6'), 129.1 (C-4'), 127.9 (C-1'), 127.2 (C-3' C-5'), 125.9 (C-1), 123.6 (C-6), 109.3 (C-2), 107.8 (C-5), 101.5 (C-7), 60.1 (C-7'); EIMS (m/z): $334[\mathrm{M}]^{+}(1 \%), 165$ (20\%), 156 (18\%), 154 (22\%), 149 (100\%), 121 (26 $\%), 119$ (6\%), 91 (17\%).

\section{Antibacterial activity}

The results of the antibacterial screening are displayed in Tables 1 and 2, respectively.

The molecule, $\mathbf{5 k}$, exhibited the highest activity against all the Gram-bacterial strains. Its MIC values were as $11.92 \pm 3.40,8.37 \pm 2.22,9.28 \pm$ $2.31,11.76 \pm 1.30$ and $10.30 \pm 1.63 \mu \mathrm{moles} / \mathrm{L}$ relative to $\mathrm{MIC}$ values of ciprofloxacin, the reference standard, as $9.42 \pm 1.09,8.02 \pm 2.17$, $8.11 \pm 1.32,8.88 \pm 2.00$ and $9.23 \pm 1.87$ $\mu$ moles/L. The molecules $5 \mathrm{c}, 5 \mathrm{e}$ and $\mathbf{5 j}$ showed

Table 1: Antibacterial activity (inhibition) of the synthesized compounds

\begin{tabular}{lccccc}
\hline \multirow{2}{*}{ Compound } & \multicolumn{5}{c}{ Inhibition (\%) } \\
\cline { 2 - 6 } & S. typhi (-) & E. coli (-) & P. aeroginosa (-) & B. subtilis (+) & S. aureus (+) \\
\hline $\mathbf{5 a}$ & $60.35 \pm 1.41$ & $64.31 \pm 5.00$ & $60.79 \pm 3.04$ & $59.90 \pm 5.00$ & $69.30 \pm 3.70$ \\
$\mathbf{5 b}$ & $61.29 \pm 4.94$ & $84.69 \pm 1.94$ & $52.46 \pm 3.71$ & $65.80 \pm 3.30$ & $67.95 \pm 3.55$ \\
$\mathbf{5 c}$ & $54.47 \pm 1.76$ & $81.69 \pm 1.09$ & $55.04 \pm 4.38$ & $51.55 \pm 0.95$ & $59.35 \pm 2.92$ \\
$\mathbf{5 d}$ & $72.65 \pm 1.24$ & $76.50 \pm 4.63$ & $58.42 \pm 3.42$ & $61.50 \pm 1.10$ & $72.85 \pm 5.00$ \\
$\mathbf{5 e}$ & $57.06 \pm 5.00$ & $60.81 \pm 1.19$ & $36.63 \pm 1.46$ & $53.45 \pm 4.55$ & $47.55 \pm 3.55$ \\
$\mathbf{5 f}$ & $65.59 \pm 1.47$ & $69.38 \pm 4.38$ & $50.92 \pm 2.08$ & $62.20 \pm 0.20$ & $65.20 \pm 5.00$ \\
$\mathbf{5 g}$ & $47.24 \pm 5.00$ & $66.50 \pm 1.38$ & $35.67 \pm 1.25$ & $56.27 \pm 2.34$ & $75.60 \pm 1.20$ \\
$\mathbf{5 h}$ & $61.18 \pm 4.71$ & $79.88 \pm 0.50$ & $54.71 \pm 4.63$ & $68.05 \pm 2.85$ & $51.65 \pm 4.50$ \\
$\mathbf{5 i}$ & $62.24 \pm 0.82$ & $84.69 \pm 1.06$ & $43.92 \pm 1.75$ & $59.10 \pm 1.40$ & $66.35 \pm 0.35$ \\
$\mathbf{5 j}$ & $53.24 \pm 5.00$ & $80.38 \pm 2.00$ & $46.63 \pm 1.46$ & $52.60 \pm 3.20$ & $69.05 \pm 3.35$ \\
$\mathbf{5 k}$ & $70.35 \pm 3.06$ & $87.38 \pm 1.75$ & $89.33 \pm 1.42$ & $72.60 \pm 2.20$ & $85.35 \pm 1.65$ \\
$\mathbf{5 l}$ & $61.76 \pm 1.88$ & $69.44 \pm 0.44$ & $68.08 \pm 2.00$ & $55.30 \pm 1.01$ & $74.55 \pm 2.45$ \\
\hline Ciprofloxacin & $\mathbf{9 1 . 1 9 \pm 2 . 1 0}$ & $\mathbf{9 0 . 4 4 \pm 1 . 2 3}$ & $\mathbf{9 2 . 0 0 \pm 2 . 7 6}$ & $\mathbf{8 9 . 9 8} \pm \mathbf{2 . 0 7}$ & $\mathbf{9 2 . 2 1 \pm 1 . 5 9}$ \\
\hline
\end{tabular}

Table 2: MIC values of antibacterial activity

\begin{tabular}{lccccc}
\hline & \multicolumn{5}{c}{ MIC } \\
\hline Compound & S. typhi $(-)$ & E. coli (-) & P. aeroginosa (-) & B. subtilis (+) & S. aureus (+) \\
\hline $\mathbf{5 a}$ & $13.03 \pm 2.07$ & $13.54 \pm 2.14$ & $14.25 \pm 2.44$ & $15.01 \pm 2.76$ & $11.58 \pm 1.40$ \\
$\mathbf{5 b}$ & $12.36 \pm 1.68$ & $8.54 \pm 4.40$ & $14.58 \pm 3.37$ & $12.06 \pm 1.15$ & $13.84 \pm 2.67$ \\
$\mathbf{5 c}$ & $17.08 \pm 1.13$ & $10.66 \pm 2.06$ & $17.79 \pm 1.94$ & $17.11 \pm 1.75$ & $13.41 \pm 2.97$ \\
$\mathbf{5 d}$ & $13.91 \pm 1.20$ & $10.86 \pm 2.11$ & $16.72 \pm 1.75$ & $13.03 \pm 1.87$ & $10.59 \pm 1.76$ \\
$\mathbf{5 e}$ & $16.68 \pm 2.40$ & $15.13 \pm 1.11$ & - & $15.51 \pm 2.98$ & - \\
$\mathbf{5 f}$ & $13.78 \pm 1.98$ & $11.67 \pm 1.22$ & $17.38 \pm 2.69$ & $12.00 \pm 2.38$ & $12.53 \pm 2.53$ \\
$\mathbf{5 g}$ & - & - & - & - & - \\
$\mathbf{5 h}$ & $12.68 \pm 2.13$ & $10.57 \pm 2.83$ & $18.08 \pm 2.05$ & $10.72 \pm 1.55$ & $16.88 \pm 1.55$ \\
$\mathbf{5 i}$ & $11.46 \pm 3.73$ & $9.23 \pm 1.28$ & - & $13.83 \pm 1.87$ & $12.44 \pm 1.90$ \\
$\mathbf{5 j}$ & $17.51 \pm 4.80$ & $10.93 \pm 1.50$ & - & $15.27 \pm 1.38$ & $10.52 \pm 1.81$ \\
$\mathbf{5 k}$ & $11.92 \pm 3.40$ & $8.37 \pm 2.22$ & $9.28 \pm 2.31$ & $11.76 \pm 1.30$ & $10.30 \pm 1.63$ \\
$\mathbf{5 l}$ & $13.89 \pm 2.00$ & $11.98 \pm 1.39$ & $11.58 \pm 3.25$ & $17.02 \pm 2.08$ & $13.83 \pm 4.07$ \\
\hline Ciprofloxacin & $\mathbf{9 . 4 2} \pm \mathbf{1 . 0 9}$ & $\mathbf{8 . 0 2} \pm \mathbf{2 . 1 7}$ & $\mathbf{8 . 1 1} \pm 1.32$ & $\mathbf{8 . 8 8} \pm 2.00$ & $\mathbf{9 . 2 3} \pm 1.87$ \\
\hline
\end{tabular}

Note: Minimum inhibitory concentration (MIC) was measured with suitable dilutions (5-30 $\mu \mathrm{g} / \mathrm{well})$ and results were calculated using EZ-Fit Perrella Scientific Inc. Amherst USA software 
$50 \%$ inhibitory action, relative to ciprofloxacin, against S. typhi; 5 e against $E$. coli; 5 a, 5 e \& 5 I against $B$. subtilis; and $\mathbf{5 h}$ against $S$. aureus. The synthesized molecules showed very low activity against $P$. aeroginosa but $\mathbf{5 k}$ and $5 \mathbf{l}$ remained good with MIC values of $9.28 \pm 2.31$ and $11.58 \pm$ $3.25 \mu \mathrm{moles} / \mathrm{L}$ respectively relative to the reference with MIC value of $8.11 \pm 1.32$ $\mu$ moles/L.

\section{DISCUSSION}

Compound $\mathbf{5 a}$, was synthesized as white amorphous solid in $92 \%$ yield with melting point of $210{ }^{\circ} \mathrm{C}$. Its molecular formula was $\mathrm{C}_{14} \mathrm{H}_{12} \mathrm{~N}_{2} \mathrm{O}_{5} \mathrm{~S}$, based on molecular ion peak at $\mathrm{m} / \mathrm{z} 320$ in EIMS and integration of protons in $1 \mathrm{H}-\mathrm{NMR}$ spectrum. In the IR spectrum, the characteristic stretching absorption bands appeared at 3256 and $1413 \mathrm{~cm}^{-1}$ assigned to $\mathrm{N}$ $\mathrm{H}$ and $\mathrm{S}=\mathrm{O}$ respectively. The prominent peaks in EIMS at $\mathrm{m} / \mathrm{z} 149$ for 3,4-methylenedioxy benzoyl and 77 for phenyl cations support the structure of 5a. Mass fragmentation of $\mathbf{5 g}$ is elaborated in Figure 1. The aromatic section of ${ }^{1} \mathrm{H}-\mathrm{NMR}$ spectrum showed three signals, one doublet and two triplets, at $\delta 7.92\left(\mathrm{~d}, \mathrm{~J}=7.6 \mathrm{~Hz}, 2 \mathrm{H}, \mathrm{H}-2^{\prime}\right.$ \& $\left.6^{\prime}\right), 7.58\left(\mathrm{t}, \mathrm{J}=7.6 \mathrm{~Hz}, 1 \mathrm{H}, \mathrm{H}-4^{\prime}\right)$ and $7.47(\mathrm{t}, \mathrm{J}=$ $7.2 \mathrm{~Hz}, 2 \mathrm{H}, \mathrm{H}-3^{\prime}$ \& 5') for five protons of phenyl group. The three aromatic protons of 1,3benzodioxol moiety resonated at $\delta 7.19(\mathrm{dd}, \mathrm{J}=$ 8.4, $1.6 \mathrm{~Hz}, 1 \mathrm{H}, \mathrm{H}-6), 7.04(\mathrm{~d}, \mathrm{~J}=1.2 \mathrm{~Hz}, 1 \mathrm{H}, \mathrm{H}-$ 2) and $6.80(\mathrm{~d}, \mathrm{~J}=8.0 \mathrm{~Hz}, 1 \mathrm{H}, \mathrm{H}-5)$; and two non-aromatic protons appeared at $\delta 6.01(\mathrm{~s}, 2 \mathrm{H}$, $\mathrm{H}-7$ ).

The whole molecular structure was also supported by twelve signals resonating in ${ }^{13} \mathrm{C}$ NMR (BB and DEPT) spectrum for five quaternary, eight methine and one methylene carbon. The four quaternary, three methine and one methylene signals of 1,3-benzodioxol-5ylcarbonyl moiety resonated at $\delta 170.5(\mathrm{C}-8)$, 148.7 (C-4), 146.4 (C-3), 125.6 (C-1), 122.6 (C6), 109.3 (C-2), 107.4 (C-5) and 101.6 (C-7). The phenyl group attached to electron withdrawing sulfono group was characterized by four signals, one for quaternary and three for methine carbons, two signals resonated with double intensities at $\delta 127.1$ (C-3' \& C-5') and 124.8 (C2 ' \& C-6') and two with single intensities at $\delta$ $145.8\left(\mathrm{C}-1^{\prime}\right)$ and 132.4 (C-4'). The above discussion corroborated the structural formula of 5a, named, 2-(1,3-benzodioxol-5-ylcarbonyl) benzenesulfonohydrazide.

The better activity of $\mathbf{5 k}$ may be attributed to the presence of trisubstituted phenyl ring linked with sulfamoyl group. In addition to $\mathbf{5 e}, \mathbf{5 g}$ remained inactive against $P$. aeruginosa $\& S$. aureus and 5i \& 5j showed no activity against $P$. aeruginosa. Nearly all the synthesized compounds exhibited activity comparable to the reference standard, ciprofloxacin.

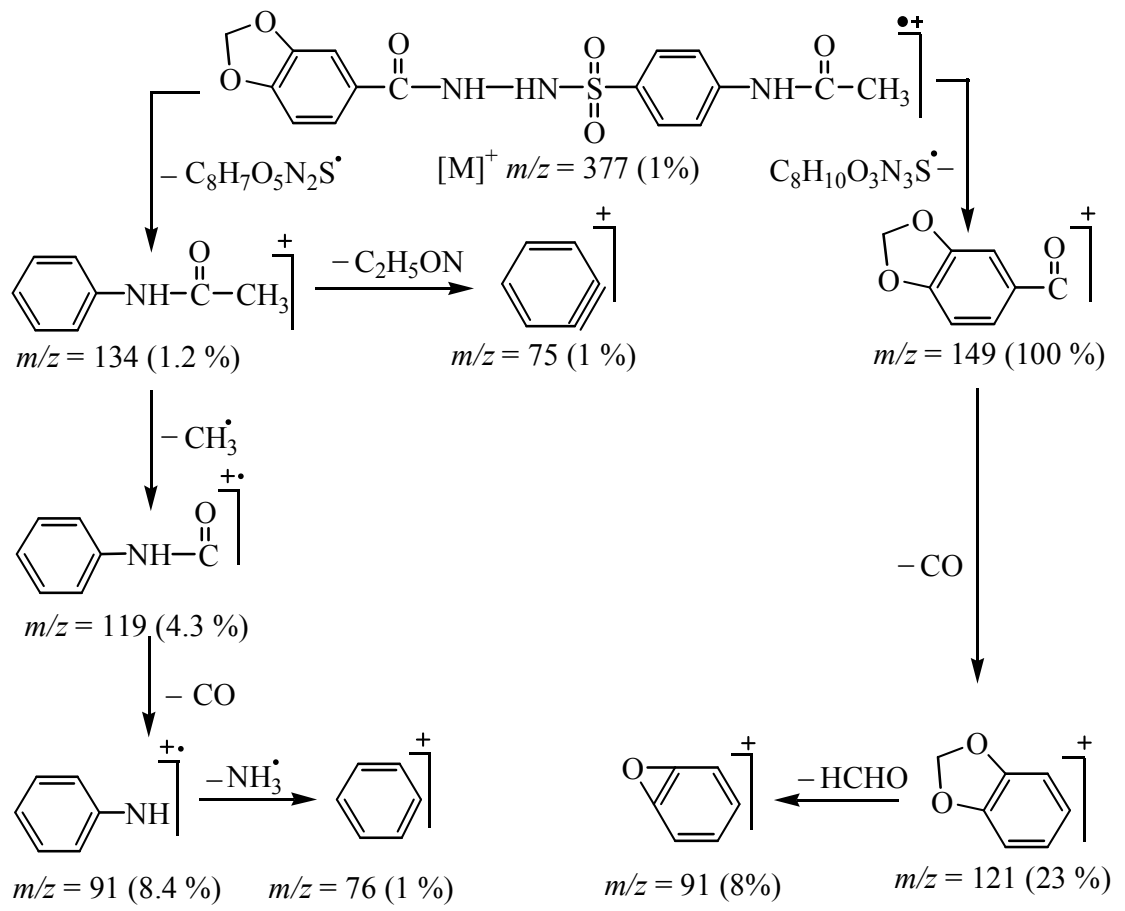

Figure 1: Mass fragmentation of 2-(1,3-Benzodioxol-5-ylcarbonyl)-4-acetamidobenzenesulfonohydrazide (5g) 


\section{CONCLUSION}

The simplicity of the synthesis of the compounds indicates that the compounds can be produced at relatively low cost. The moderate but significant inhibitory activities of the synthesized sulfonamides bearing the heterocyclic moiety are known to be valuable for their pharmaceutical industry. Thus, these molecules should be further evaluated as lead compounds for discovery of new drug candidates.

\section{ACKNOWLEDGEMENT}

The authors acknowledge Higher Education Commission of Pakistan for financial assistance.

\section{REFERENCES}

1. Ozbek N, Katircioglu H, Karacan N, Baykal T. Synthesis, characterization and antimicrobial activity of new aliphatic sulfonamide. Bioorgan Med Chem 2007; 15: 5105-5109.

2. Alsughayer A, Elassar AZA, Mustafa S, Al Sagheer F. Synthesis, structural analysis and antibacterial activity of new potent sulfonamide derivatives. J Biomater Nanobiotechnol 2011; 2: 144-149.

3. Kumar S, Niranjan MS, Chaluvaraju KC, Jamakhandi CM, Kadadevar D. Synthesis and antimicrobial study of some schiff bases of sulfonamides. J Curr Pharm Res 2010; 1: 39-42.

4. Baskin JM, Wang Z. A mild, convenient synthesis of sulfinic acid salts and sulfonamide from alkyl and aryl halides. Tetrahedron Lett 2002; 43: 8479-8483.

5. Sondhi SM, Dwivedi AD, Singh J, Gupta PP. Synthesis and anti-inflammatory activity evaluation of some sulfonamide and amide derivatives of 4-aryl-3-(2 or 4picolyl)-2-imino-4-thiazolines. Indian J Chem 2010; 49B: 1076-1082.

6. Cami GE, Arellano RD, Del MC, Fustero S, Pedregosa JC. Synthesis, growth and characterization of new 1 , 3, 4-thiadiazole-5-( $\mathrm{N}$-substituted)-sulfonamides crystals. J Argent Chem Soc 2006; 94: 5-17.

7. Argyropoulou I, Geronikaki A, Vicini P, Zani F. Synthesis and biological evaluation of sulfonamide thiazole and benzothiazole derivatives as antimicrobial agents. Arkivoc. 2009; 6: 89-102.

8. Seikwan O, Moon HI, Son IIH, Jung JC. Synthesis of sulfonamides and evaluation of their histone deacetylase (HDAC) activity. Molecules 2007; 12: 1125-1135.

9. Shi F, Tse MK, Zhou S, Pohl MM, Radnik J, Huebner S, Jaehnisch K, Brueckner A, Beller M. Green and efficient synthesis of sulfonamides catalyzed by nano-Ru/Fe3O4. J Am Chem Soc 2009; 131: 17751779.

10. Thakur A, Thakur M, Khadikar PV. QSAR study on inhibition of E. coli by sulfonamide. Arkivoc 2006; 14: 87-102.

11. Aziz-ur-Rehman, Siddiqa A, Abbasi MA, Rasool S, Siddiqui SZ, Gul S, Ashraf M, Nasar R. Enzyme inhibition studies on $\mathrm{N}$-substituted sulfonamides derived from m-Phenetidine. Pak J Chem 2013; 3(3): 1-7.

12. Kaspady M, Narayanaswamy VK, Raju M, Rao GK. Synthesis, antibacterial activity of 2,4-disubstituted oxazoles and thiazoles as bioesters. Lett Drug Des Discov 2009; 6: 21-28.

13. Yang CR, Zang Y, Jacob MR, Khan SI, Zhang YJ, Li XC. Antifungal activity of $C-27$ steroidal saponins. Antimicrob Agents Ch 2006; 50(5): 1710-1714. 